\title{
sciendo
}

DOI: 10.1515/aon-2019-0005

\section{EDAS (EGNOS DATA ACCESS SERVICE) DIFFERENTIAL GNSS CORRECTIONS: A RELIABLE FREE-OF-CHARGE ALTERNATIVE FOR PRECISION FARMING IN EUROPE}

\author{
J. Vázquez, E. Lacarra, J. Morán, M.A. Sánchez, A. González, J. Bruzual \\ ESSP SAS, \\ J. Rioja, Topcon Precision Agriculture Europe
}

\begin{abstract}
EDAS (EGNOS Data Access Service) is the EGNOS internet broadcast service, which provides free of charge access to the data collected and generated by the EGNOS infrastructure. EDAS disseminates over the Internet, both in real time and via an FTP archive, the raw data of the GPS, GLONASS (no commitment on GLONASS data is provided (1)) and EGNOS GEO satellites collected by the receivers located at the EGNOS reference stations, which are mainly distributed over Europe and North Africa. The EDAS services offer several types of GNSS data in various protocols and formats, such as DGNSS corrections. This paper reports on the results of some in-field tests conducted by ESSP and Topcon Agriculture to confirm the suitability of EDAS DGNSS corrections for precision farming in Europe.
\end{abstract}

The European Commission (EC) is the owner of EGNOS system (including EDAS) and has delegated the exploitation of EGNOS to the European GNSS Agency (GSA). EDAS service provision is performed by ESSP, as EGNOS Services Provider, under contract with the GSA, the EGNOS program manager.

In the ENC 2018 article "EDAS (EGNOS Data Access Service): Differential GPS corrections performance test with state-of-the-art precision agriculture system", ESSP and Topcon Agriculture presented the results of the first in-field test conducted in a dynamic and real-life environment in the summer of 2017. The test results indicated that the EDAS DGNSS corrections could enable a reliable pass-to-pass accuracy performance for a wide range of precision agriculture applications and become an attractive solution for cereal farms, when the farm is located in the vicinity of an EGNOS reference station. In particular, Topcon Agriculture acknowledged that the observed performance was sufficient to support the following precision agriculture applications: spraying and spreading of any crop type, tilling and harvesting of cereal.

Then, ESSP and Topcon Agriculture engaged in additional testing activities to further characterise the EDAS DGPS performance in different scenarios (i.e. at various European locations and with a variety of distances between the designated farm and the target EGNOS reference station).

In each test, multiple runs with the rover tractors have been performed over the reference patterns predefined in the Topcon guidance systems. Data recorded during the tests has been analysed in detail, looking at the key performance indicators (e.g. cross track error and pass-to-pass performance) that characterize the EDAS DGPS performance for precision agriculture applications. Different techniques for the computation of the pass-to-pass accuracy performance have been used, including a procedure to measure live in the field and a post-processing alternative. The diversity of scenarios available allows drawing conclusions on the applicability of EDAS DGPS corrections (in terms of maximum distance from the target EGNOS station) for precision agriculture and also 
understanding the impact of operationally relevant aspects such as the quality of the mobile internet coverage (highly variable across Europe).

The EDAS system and its architecture, the main types of data disseminated through EDAS services and the online information available to the EDAS users are introduced in this paper. In particular, the EDAS Ntrip service is described in detail, since it provides the differential corrections to the GPS and GLONASS satellites at the EGNOS reference stations in RTCM format, which are the basis for the present study.

The article also reports on the results of the latest tests, which have been performed using Topcon receivers, vehicles and auto-steering systems. In all cases, two different Topcon guidance systems on board tractors were running simultaneously to assess the EDAS DGPS positioning performance with respect to a the reference provided by a top-performing RTK-based Topcon solution.

The objective of this paper is to draw conclusions on the use of EDAS DGPS corrections as a reliable freeof-charge alternative for precision farming in Europe (especially for cereal farms), based on the available performance results from the testing campaign and the feedback from the involved precision agriculture experts.

Keywords - GNSS, Galileo, EDAS, tracking, firefighting, multisensor, fusion, communication optimization

\section{INTRODUCTION TO EDAS}

EGNOS, the European Satellite Based Augmentation System (SBAS), currently provides corrections and integrity information to GPS signals over Europe and is fully interoperable with other existing SBAS systems (e.g. WAAS, the North American SBAS).

ESSP SAS (European Satellite Services Provider) is the EGNOS system operator and EGNOS Service provider, under contract with the European GNSS Agency (GSA), for the following three services:

- EGNOS Safety of Life (SoL) Service, for safety-critical transport applications, including civil aviation, which require enhanced and guaranteed performance and an integrity warning system ([2]).

- EGNOS Open Service (OS), free and open to the public, which provides positioning, navigation and timing services to be used by mass-market receivers and common user applications ([3]).

- EGNOS Data Access Service (EDAS), which is intended for users who require enhanced performance or products for commercial and professional use ([1]).

EDAS gathers the raw data coming from the GPS, GLONASS and EGNOS GEO satellites collected by the receivers located at the EGNOS stations. The EGNOS operational system comprises 39 Ranging and Integrity Monitoring Stations ( RIMS) and 4 Navigation Land Earth Stations ( NLES), mainly distributed over Europe and North Africa. EDAS reformats and disseminates EGNOS GNSS data in real time and through an FTP archive to EDAS users and/or Service Providers.

The following table summarizes the types of data that can be retrieved via the different EDAS services: 
Table 1: EDAS Services.

\begin{tabular}{|c|c|c|c|c|c|c|c|}
\hline \multirow[b]{2}{*}{ 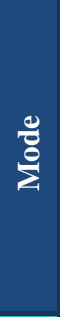 } & \multirow[b]{2}{*}{$\begin{array}{c}\text { EDAS } \\
\text { Service }\end{array}$} & \multicolumn{4}{|c|}{ Type of Data } & \multirow[b]{2}{*}{ Protocol } & \multirow[b]{2}{*}{ Formats } \\
\hline & & 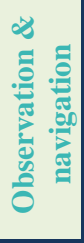 & 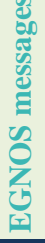 & 约 & 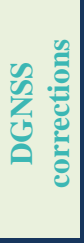 & & \\
\hline \multirow{4}{*}{ ב } & SL/DF 0 & $\mathrm{X}$ & $X$ & & & EDAS & ASN.1 \\
\hline & SL/DF 2 & $\mathrm{X}$ & $\mathrm{X}$ & & & EDAS & RTCM 3.1 \\
\hline & SISNeT & & $X$ & & & SISNeT & $\begin{array}{c}\text { RTCA } \\
\text { DO-229D }\end{array}$ \\
\hline & Ntrip & $X$ & & $X$ & $\mathrm{X}$ & Ntrip v1.0, v2.0 & $\begin{array}{c}\text { RTCM } \\
2.1,2.3,3.1 \\
\end{array}$ \\
\hline : & FTP & $X$ & $X$ & & & FTP & $\begin{array}{l}\text { RINEX 2.11, RINEX B 2.10, EMS, } \\
\text { IONEX, SL0 and SL2 raw binary data }\end{array}$ \\
\hline
\end{tabular}

With regards to the EDAS NTRIP service, which is the EDAS service that has been used for the tests addressed in the present paper, an important update was implemented in July 2018, aiming at maximizing the number of users that can benefit from this EDAS service ([18]). Until mid-July 2018, the EDAS Ntrip service was based on version 2.0 ([10]) of Ntrip protocol over RTSP/RTP. Now, the upgraded EDAS Ntrip service supports the different Ntrip protocol versions/options (Ntrip v1, Ntrip v2 over HTTP and Ntrip v2 over RTSP/RTP); in other words, compatibility with the user equipment is now ensured.

For more information about EDAS, please, refer to the EDAS Service Definition Document ([1]) and the EGNOS User Support Website, https://egnos-user-support.essp-sas.eu/.

\section{EDAS FOR DGNSS POSITIONING}

\section{EDAS DGNSS overview}

Differential GNSS (DGNSS) corrections are sent through the EDAS Ntrip Service via Internet to support applications requiring sub-meter accuracies.

The EGNOS Stations (RIMS and NLES) are static reference receivers, which are placed at fixed and known surveyed locations. Then, since the GNSS satellite positions and the reference antenna locations from the EGNOS stations are known, the ranges (distance from satellite to station) can be determined precisely. By comparing these ranges to those obtained from the satellite observation measurements, the pseudorange errors can be accurately estimated (i.e. ionospheric delays, tropospheric delays, ephemeris errors and satellite clock errors), and corrections determined. These DGNSS corrections can then be broadcast to nearby users, who apply them to improve their position solutions.

The DGNSS corrections are sent through the EDAS Ntrip Service in RTCM 2.1 ([9]) and RTCM 2.3 ([10]) formats, using the messages shown in table below: 
Table 2: EDAS DGNSS Message Types.

\begin{tabular}{|l|l|l|}
\hline \multirow{2}{*}{\multicolumn{1}{|c|}{ EDAS DGNSS Messages }} & \multicolumn{2}{c|}{ Message Types } \\
\cline { 2 - 3 } & \multicolumn{1}{c|}{ RTCM 2.1 } & RTCM 2.3 \\
\hline Differential GPS Corrections & 1 & 1 \\
\hline GPS Reference Station Parameters & 3 & 3 \\
\hline Reference Station Datum & N/A & 4 \\
\hline Extended Reference Station Parameters & N/A & 22 \\
\hline Antenna Type Definition Record & N/A & 23 \\
\hline Antenna Reference Point (ARP) & N/A & 24 \\
\hline Differential GLONASS Corrections & N/A & 31 \\
\hline GLONASS Reference Station Parameters & N/A & 32 \\
\hline
\end{tabular}

For detailed information about the connection and usage of the EDAS Ntrip service, the EDAS Ntrip User Information Package ([7]) is provided upon registration to EDAS.

\section{Pass-to-pass (P2P) accuracy concept}

In the agriculture domain, the pass-to-pass accuracy is the key performance indicator to assess the precision of guidance systems. It characterizes the short-term dynamic performance determined from off-track errors along the straight segment passes (error with respect to the desired path - blue and grey lines in Figure 1 - in the direction perpendicular to the tractor trajectory). In addition to a sufficient absolute horizontal accuracy (at least 1 meter -95th percentile- is required for cereal and dry soil cultivation), the stability of the errors affecting the position solutions can have large economic impacts. In order to cover a given field, farmers typically define a pattern which is composed by a set of parallel lines separated by the implement distance. In this manner, if the guidance system precisely follows the reference pattern, the efficiency and productivity of operations is maximized ensuring that the same soil is not covered twice and avoiding that a certain part of the field is not treated.

\section{Previous work and motivation}

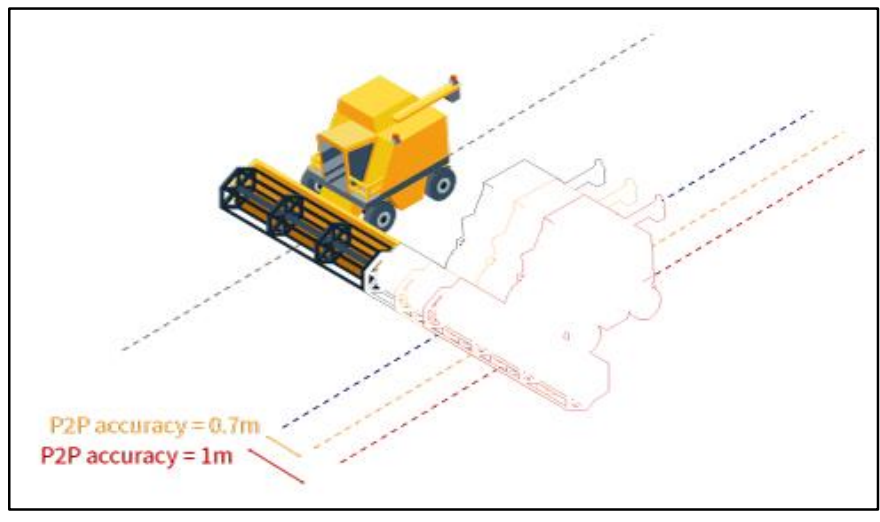

Figure 1: pass-to-pass (P2P) concept ([17]).

In the related article presented at the ENC 2018 ([15]), ESSP and Topcon Agriculture presented the results of the first in-field test conducted in a dynamic and real-life environment in the summer of 2017. The tests results indicated that the EDAS DGPS corrections could enable a reliable pass-to-pass accuracy performance for a wide range of precision agriculture applications and become an attractive solution for cereal farms, when the farm is located in the vicinity of an EGNOS reference station. In particular, Topcon Agriculture acknowledged that the observed performance was sufficient to support the following precision agriculture applications: spraying and spreading of any crop type, tilling and harvesting of cereal. In order to confirm the 
preliminary conclusions from the first test, additional tests have been done by Topcon Agriculture and ESSP to verify the adequacy of EDAS DGPS corrections for precision agriculture in different European locations and covering different scenarios from the point of view of the distance of the test location from the selected EGNOS reference station (i.e. using different baselines).

The results of all the tests, which have been performed using Topcon receivers, vehicles and auto-steering systems, are presented in the following sections. In all cases, two different Topcon guidance systems on board tractors were running simultaneously to assess the EDAS DGPS positioning performance with respect to the reference provided by a top-performing RTK-based Topcon solution.

\section{EDAS DGPS For PRECISION AgRiculture: TeSt CAMPAIGN}

\section{Test scenarios}

Three dynamic test campaigns have been conducted by Topcon Agriculture and ESSP. Considering the geographical location of the farms, and in order to have a variety of baselines, the following EGNOS stations were selected as reference for the tests:

\begin{tabular}{|c|c|c|c|}
\hline \multicolumn{5}{|c|}{ Table 3: Test locations and selected reference station. } \\
\hline Farm Location & Date & EGNOS Station & Baseline (km) \\
\hline Lisbon area (PT) & $13 / 06 / 2017$ & Lisbon & $40 \mathrm{~km}$ \\
\hline Seville area (SP) & $11 / 09 / 2017$ & Malaga & $110 \mathrm{~km}$ \\
\hline \multirow{2}{*}{ York area (UK) } & $29 / 11 / 2017$ & Glasgow & $320 \mathrm{~km}$ \\
\cline { 2 - 4 } & & Swanwick & $340 \mathrm{~km}$ \\
\hline
\end{tabular}

Although no implement (i.e. tool to perform a specific agriculture task) was attached to the tractor for the tests, a theoretical implement was configured in the X35 consoles for all tests. This means that, in the best case and as the different lines are covered with the tractor, the actual lateral separation between consecutive lines should match the configured implement width.

\section{Test set-up}

For the three tests described above, a tractor was equipped with two different Topcon guidance systems running simultaneously in order to be able to assess the EDAS DGPS positioning performance with respect to the reference, which was obtained from a top-performing RTK-based Topcon solution (HiperV RTK base) that typically provides 1-2 $\mathrm{cm}$ horizontal accuracy.

Hence, two independent positioning outputs were continuously available (receivers were placed along the same longitudinal axis on the roof of the tractor):

- RTK position: provided by the AGI-4 receiver fed by Topcon's HiperV RTK base.

- DGPS position: provided by the AGI-4 receiver fed by the EDAS Ntrip service.

On board the tractor, 2 Topcon X35 consoles were installed, each one connected to one of the receivers. Additionally, a Topcon AES-25 electric steering system was installed on the tractor so that the selected navigation input (either the RTK or the EDAS DGPS input) could be used to automatically guide the tractor along the defined reference pattern (see Figure 2). 

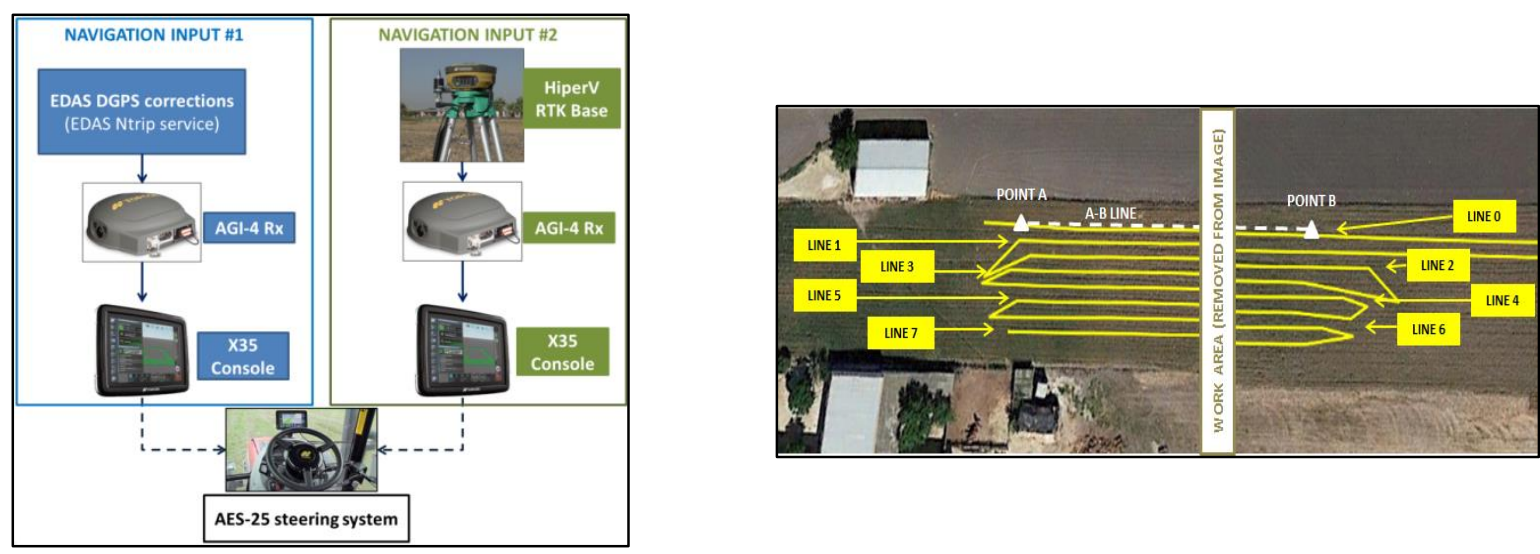

Figure 2: Navigation inputs used for the tests (left); farm field and pattern lines in Seville, Spain (right).

In order to define the reference pattern, the tractor was placed on one side of the farm (Point A in Figure 2). After marking that point in both X35 Consoles simultaneously, the tractor followed (manually guided) a straight line parallel to the edge of the farm. At a distance of approximately 200 metres from Point A, the second reference point was defined (Point B in Figure 2). The imaginary straight line from Point A to Point B became our line \#0. Then, the parallel lines automatically defined by the X35 Console with a lateral separation equal to the configured implement width ( 3 metres in our case) completed the definition of the pattern to be used for the tests.

During the whole test duration, the logging function of the X35 Consoles was active so that, on top of the measurements taken in-field, an exhaustive data analysis could be performed after the tests.

\section{Procedures for pass-to-pass accuracy assessment}

On top of the information displayed by the X35 Consoles, which provide an estimated deviation with respect to the reference pattern in real-time, two different approaches have been used to measure the pass-to-pass accuracy:

- Post-processing: the data logged by the X35 Consoles was post-processed to compute the cross track error of the EDAS DGPS solution with respect to the RTK one (reference) along the pattern.

The positions reported by both X35 Consoles (each fed by one AGI-4 receiver) were post-processed to compute the instantaneous cross track error of the EDAS DGPS solution with respect to the RTK one (i.e. difference between sensor outputs in transversal direction to the corresponding line). After that, the instantaneous pass-to-pass error along the corresponding line ("line" and "pass" are used to refer to the same concept: a straight segment along the work field) for any given couple of consecutive lines is obtained by subtracting the instantaneous cross track errors of the current line and the previous one. Figure 3 illustrates this process.

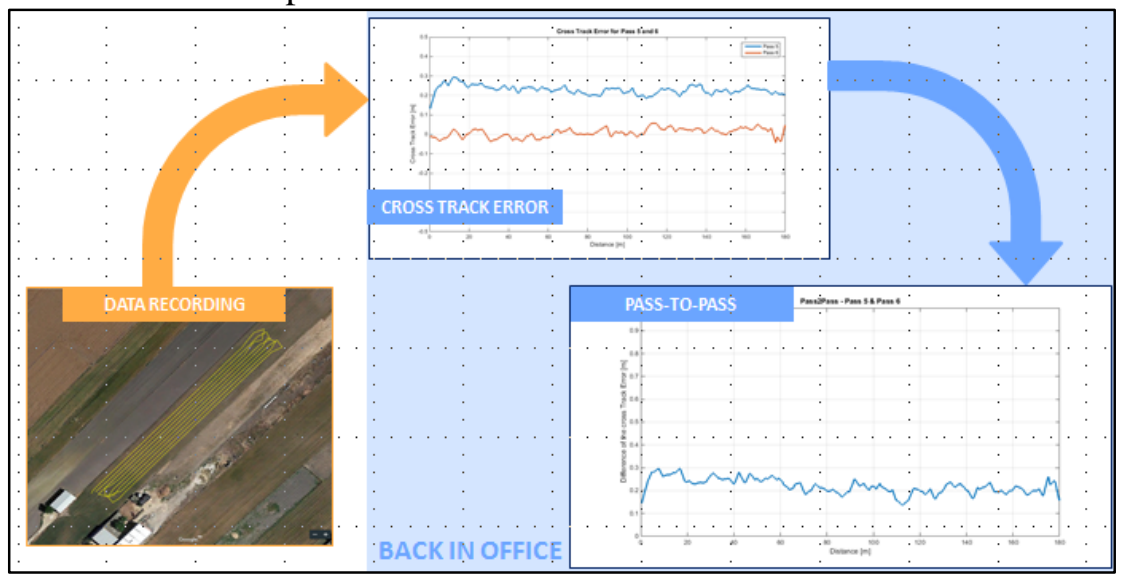

Figure 3: Instantaneous pass-to-pass performance computation (post-processing). 
- In field measurements: the lateral separation between consecutive lines was also manually measured in field.

The process for the in-field measurement of the pass-to-pass accuracy for a given couple of lines is depicted in Figure 4 and consists on the following steps:

- Step 1: The tractor, with the steering system engaged to the EDAS DGPS navigation input, is stopped at a designated area (Measurement Area in Figure 4) within a given line. Using a physical point of the tractor's external bodywork, a first mark is done on the ground (Orange $X$ in Figure 4).

- Step 2: With the steering function engaged to the EDAS navigation system output, the desired line length is covered by the tractor (Line 0 in Figure 4).

- Step 3: At the end of the line, the tractor is manually turned to the next line (Line 1 in Figure 4) and the steering system is engaged again to the EDAS navigation system input.

- Step 4: Once the tractor has covered the full line in the opposite direction as the previous one, the tractor is stopped at the Measurement area. Then, using the same physical reference of the tractor's external bodywork as in step 1, a second mark is done (Blue X in Figure 4); this second mark needs to be aligned with the first mark (Orange $X$ in Figure 4) perpendicularly to the subject lines.

- Step 5: The distance in the transversal direction to concerned lines is measured. The deviation of this measurement from the configured implement width (target) is the pass-to-pass accuracy for the concerned lines - Line 0 and Line 1 in Figure 4).

- Step 6: Then, the process is restarted (Step 1) for the next couple of lines.

\section{Test Results in Marchena, Spain (110 km baseline)}

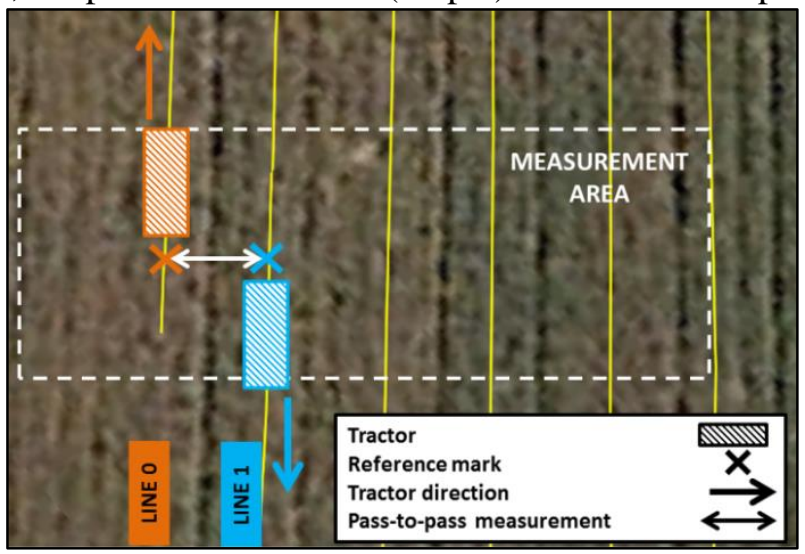

Figure 4: In-field pass-to-pass measurement (procedure).

The results from the test in Marchena were already reported at the ENC 2018 ([15]). Here, just a summary of the main results is presented; for a more exhaustive description of the test results, please refer to ([15]).

Three different runs covering the seven available lines due to the farm dimensions were done in the Marchena test campaign. For the first two runs, the pass-to-pass performance has been computed by post-processing the data logged by the X35 Consoles, while in the third run, in-field measurements were taken.

Table 4 summarises the main results obtained during this test.

Table 4: Marchena EDAS DGPS pass-to-pass accuracy

\begin{tabular}{|c|c|c|c|c|c|c|c|}
\hline \multirow{2}{*}{ Run } & \multicolumn{6}{|c|}{ pass-to-pass accuracy average (cm) / Lines } \\
\cline { 2 - 8 } & $\mathbf{1 - 2}$ & $\mathbf{2 - 3}$ & $\mathbf{3 - 4}$ & $\mathbf{4 - 5}$ & $\mathbf{5 - 6}$ & $\mathbf{6 - 7}$ & All \\
\hline 1 (post-processsing) & 20 & 14 & 16 & 28 & 22 & - & $\mathbf{2 1}$ \\
\hline $\mathbf{2}$ (post-processsing) & 23 & 20 & 18 & 19 & 22 & 19 & $\mathbf{2 2}$ \\
\hline $\mathbf{3}$ (in-field) & $3-4$ & $10-12$ & - & $1-3$ & - & $7-8$ & $\mathbf{5 - 7}$ \\
\hline
\end{tabular}

\section{Test Results in Lisbon (40 km baseline)}

For this scenario, the tractor's automatic steering system was always fed by the position provided by the AGI-4 receiver using the EDAS DGPS corrections from the EGNOS Station in Lisbon, approximately $40 \mathrm{~km}$ away from the farm. 
Five different runs with different patterns in each run were done. For instance, an example of one of the patterns used in this test is shown in Figure 5. For this run, the pass-to-pass accuracy computed by postprocessing the logs reported by the onboard X35 consoles was $10 \mathrm{~cm}$ on average for all the passes. The instantaneous pass-to-pass error obtained during this run is depicted in Figure 5.
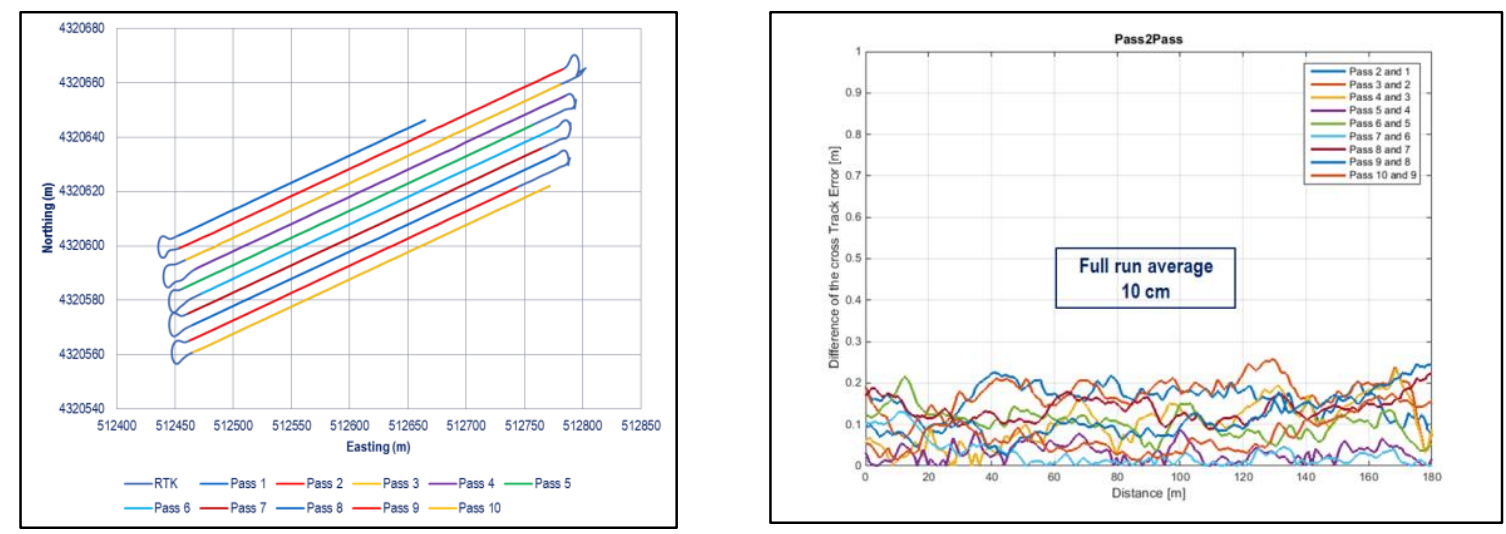

Figure 5: Lisbon pattern-left- and instantaneous pass-to-pass accuracy-right-for Run\#4.

On top of the results obtained by post-processing the information logged by the X35 consoles, in-field measurements were also done for the $4^{\text {th }}$ run.

Table 5: Lisbon EDAS DGPS pass-to-pass accuracy

\begin{tabular}{|c|c|c|c|c|c|c|c|c|c|c|c|c|c|c|}
\hline \multirow[b]{2}{*}{ Run } & \multicolumn{14}{|c|}{ pass-to-pass average $(\mathrm{cm}) /$ Lines } \\
\hline & $1-2$ & $2-3$ & 3-4 & 4-5 & $5-6$ & $7-8$ & 8-9 & $9-10$ & 10-11 & 11-12 & $12-13$ & 13-14 & 14-15 & All \\
\hline 1 & 4 & 6 & 6 & 2 & 6 & 8 & 8 & 3 & 13 & 5 & 13 & - & - & 7 \\
\hline 2 & 10 & 9 & 4 & 13 & 3 & 7 & - & - & - & - & - & - & - & 8 \\
\hline 3 & 6 & 8 & 11 & 24 & 13 & 12 & 4 & 4 & 6 & - & - & - & - & 10 \\
\hline 4 & 16 & 17 & 10 & 3 & 10 & 3 & 14 & 11 & 7 & - & - & - & - & 10 \\
\hline 4 (in-field) & 13 & - & 8 & - & 14 & - & 5 & - & 3 & - & - & - & - & 9 \\
\hline 5 & 7 & 11 & 2 & 3 & 7 & 12 & 5 & 5 & 3 & 10 & 13 & 4 & 3 & 6 \\
\hline
\end{tabular}

As detailed in Table 5 and also depicted in Figure 6, the average error measured in all the passes done in Lisbon is consistently below $10 \mathrm{~cm}$ for most of the passes (only 1 above $20 \mathrm{~cm}$ ), being the total average passto-pass accuracy $8 \mathrm{~cm}$ (considering all the runs and all the passes). 


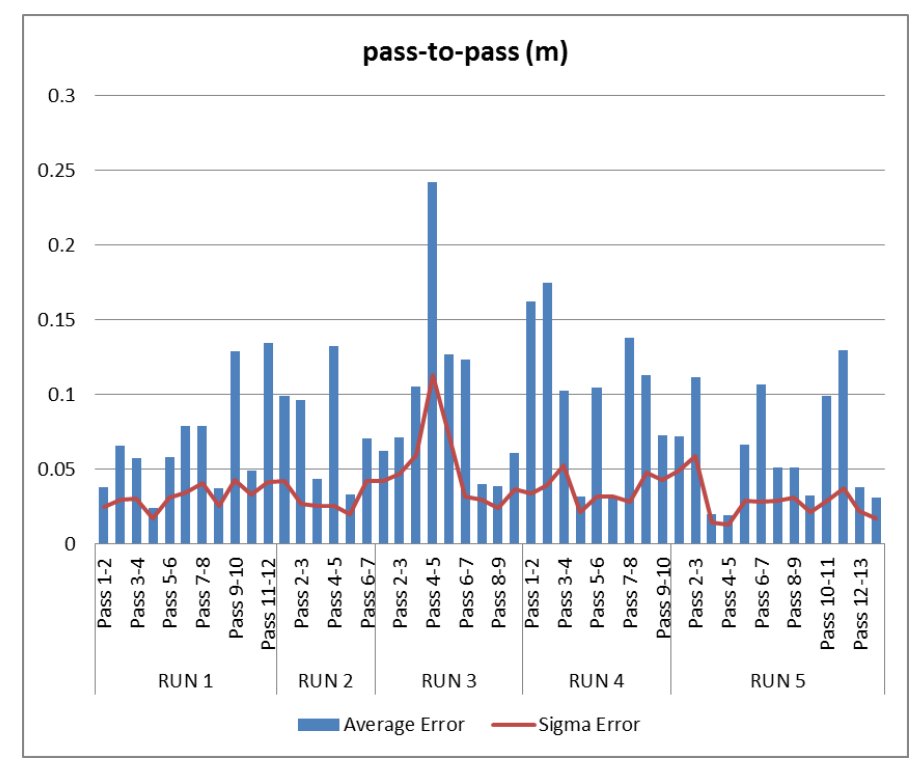

Figure 6: Lisbon EDAS DGPS pass-to-pass accuracy

One specific difficulty during the test in Lisbon was the degraded mobile internet coverage in some areas of the test farm. The main impact that could be detected on the GNSS sensor (AGI-4 receiver) was a decrease in the number of satellites used in the differential solution. Figure 7 shows the instantaneous pass-to-pass accuracy for run\#2 and run\#3, which were partially affected by this issue. In Figure 7 passes with 6 or less satellites being used for the differential solution computation are displayed in red colour while those in which more than 7 satellites were used are shown in green.

As it can been below, in Run\#3, the decrease in the number of satellites considered for the DGPS solution slightly affected the pass-to-pass accuracy, while such effect is not observed at all in Run\#2. The impact of a degraded internet connectivity needs to be further analysed (additional data needed) although, at this stage and based on the available results, it seems that, even under difficult conditions, the EDAS DGPS corrections would still deliver the performance needed for the target operations (average of $13 \mathrm{~cm}$ pass-to-pass accuracy considering only the passes with 6 or less satellites in use in Run\#3).
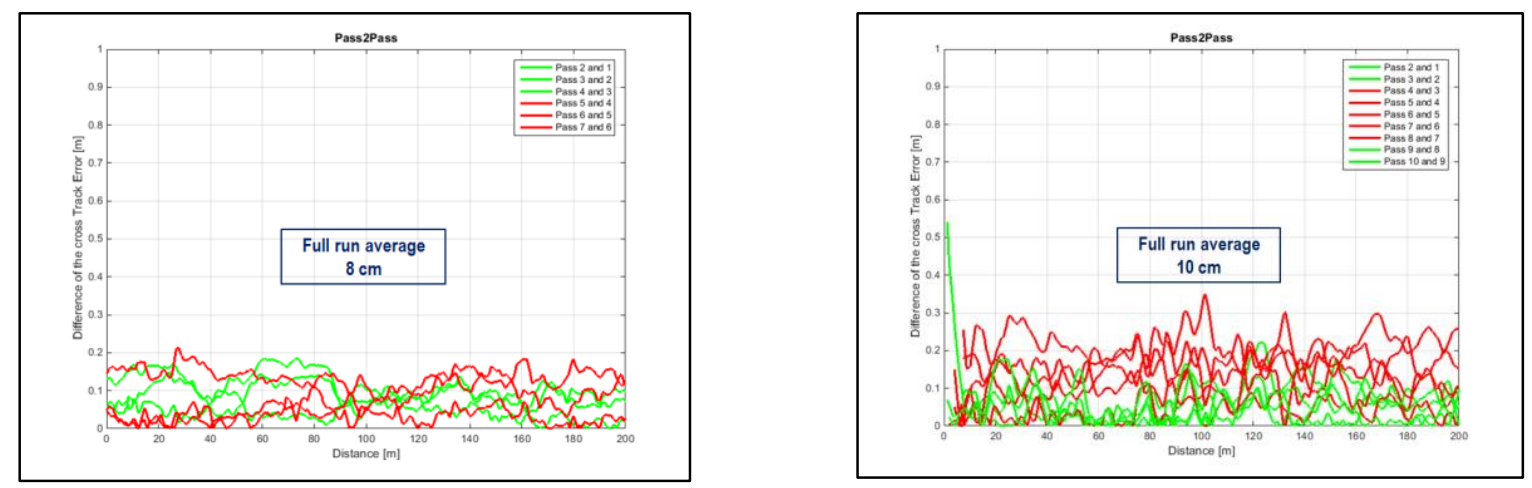

Figure 7: Instantaneous pass-to-pass accuracy - Run\#2 (left) and Run\#3 (right).

\section{Test Results in York $(\approx 330 \mathrm{~km}$ baseline $)$}

In this case, the selected test location provides a long baseline between the farm and the selected EGNOS reference station. During the first three runs, the EDAS DGPS corrections provided for the EGNOS station in Glasgow (320 km from York) were used by the AGI-4 receiver whereas in the last run, the EDAS DGPS corrections for the Swanwick station were applied (340 km from the farm).

Table 6 details the average pass-to-pass obtained in the 4 runs done in York, being the total average of the pass-to-pass accuracy $8.4 \mathrm{~cm}$. 
Table 6: York EDAS DGPS pass-to-pass accuracy

\begin{tabular}{|c|c|c|c|c|c|c|c|c|}
\hline \multirow{2}{*}{ Run } & \multicolumn{8}{|c|}{ pass-to-pass average (cm)/Lines } \\
\cline { 2 - 9 } & $\mathbf{1 - 2}$ & $\mathbf{2 - 3}$ & $\mathbf{3 - 4}$ & $\mathbf{4 - 5}$ & $\mathbf{5 - 6}$ & $\mathbf{6 - 7}$ & $\mathbf{7 - 8}$ & All \\
\hline $\mathbf{1}$ & 4 & 6 & 9 & 9 & 7 & 5 & 8 & $\mathbf{7}$ \\
\hline $\mathbf{2}$ & 9 & 6 & 5 & 7 & - & - & - & $\mathbf{8}$ \\
\hline $\mathbf{3}$ & 21 & 25 & 21 & 6 & 5 & - & - & $\mathbf{1 5}$ \\
\hline $\mathbf{4}$ & 3 & 4 & 4 & 5 & 8 & - & - & $\mathbf{5}$ \\
\hline $\begin{array}{c}\mathbf{4} \text { (in- } \\
\text { field) }\end{array}$ & 14 & 11 & 2 & 0 & 4 & - & - & $\mathbf{6}$ \\
\hline
\end{tabular}

These pass-to-pass accuracy statistics are also depicted in the following graphic (Figure 8), showing a mean pass-to-pass error in the order of 5-10 $\mathrm{cm}$ for all the passes but for the third run, when the average error goes up to 20-25 cm. Considering the large baselines tested, the $10 \mathrm{~cm}$ pass-to-pass performance level obtained in this campaign is especially remarkable even if, for one of the runs, some passes showed a pass-to-pass accuracy of $20-25 \mathrm{~cm}$.

However, as already mentioned, depending on the application, a certain level of absolute accuracy is also required. For the case of cereal and dry soil cultivation, at least 1 meter -95th percentile- is required. In relation to this, previous studies ([19]) have shown that EDAS DGPS corrections can support sub meter level accuracies for baselines of up to $260 \mathrm{~km}$ with respect to the selected EGNOS station - the resulting coverage area is shown in Figure 8-. Hence, based on the results from the test in York $(\approx 320 \mathrm{~km}$ baseline $)$, it can be assumed that, within that range, EDAS DGPS corrections can also support pass-to-pass accuracies in the order of 10-20 cm.
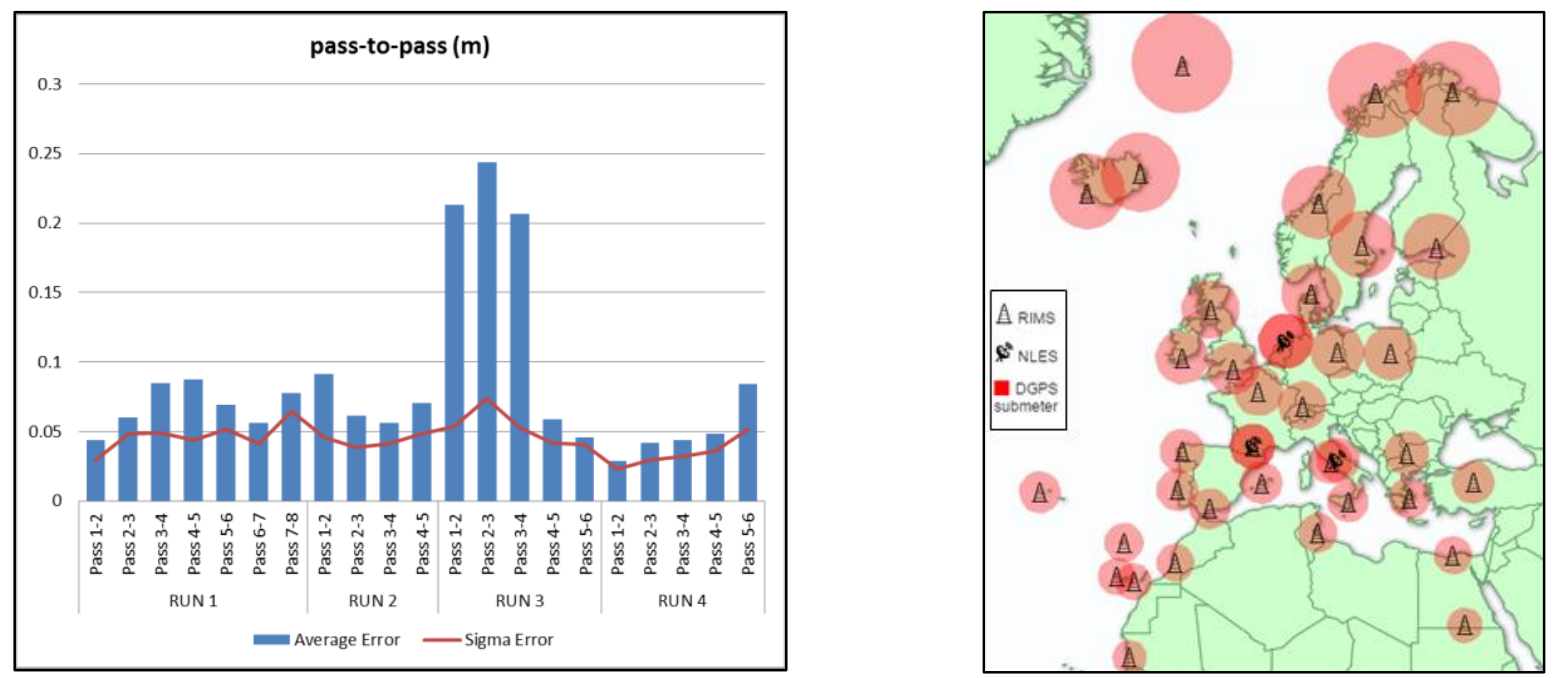

Figure 8: York EDAS DGPS pass-to-pass accuracy -left-; Expected EDAS DGPS submeter accuracy coverage ([19]) - right-.

\section{Test Results: Overview}

Overall, the results of 3 different in-field tests have been presented. As explained in the previous sections, the test scenarios were quite diverse in terms of:

- Baseline: the distance from the selected farm to the EGNOS station used as reference for the EDAS DGPS corrections ranged from $40 \mathrm{~km}$ (Portugal) to approximately $320 \mathrm{~km}$ (UK); with an intermediate scenario at $110 \mathrm{~km}$ (Spain).

- EGNOS stations used at reference: DGPS corrections from four different EGNOS stations (Lisbon, Malaga, Swanwick and Glasgow) have been used to confirm the quality of the corrections independently of the selected station. 
- Mobile internet coverage: while no issue was observed during the tests in UK and Spain, some coverage degradations were observed in the test in Portugal.

- Geographical location: tests have been conducted in Spain, Portugal and United Kingdom.

However, the above differences have not been translated into significant accuracy performance drifts and, in general terms, EDAS DGPS corrections have proven to deliver pass-to-pass accuracies below $20 \mathrm{~cm}$ consistently in all tests. Figure 9 shows the cumulative distribution of the average pass-to-pass accuracy obtained for all passes in the 3 tests, based on the results obtained in post-processing mode. From Figure 9, it is to be highlighted that the average of the instantaneous pass-to-pass accuracy remained below:

- $10 \mathrm{~cm}$, for almost $61 \%$ of the passes.

- $20 \mathrm{~cm}$, for almost $85 \%$ of the passes.

- $25 \mathrm{~cm}$, for more than $95 \%$ of the passes.

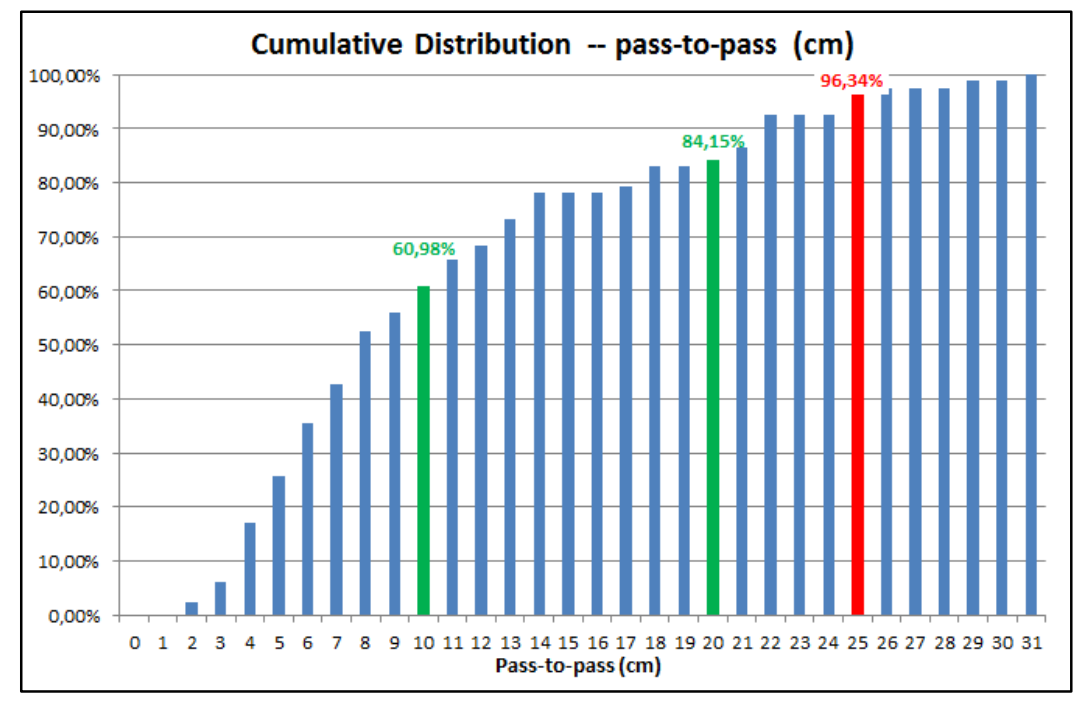

Figure 9: Cumulative distribution of the pass-to-pass accuracy (all tests)

\section{CONCLUSIONS}

The current paper is focused on the EDAS Ntrip Service, which can be used for differential positioning since it provides DGNSS corrections for the EGNOS stations, located mainly over Europe and North of Africa, in real-time over the internet.

An important update of the EDAS Ntrip service has been implemented in July 2018 which aims at maximizing the number of users that can benefit from this EDAS service ([18]). Since mid-July 2018, the EDAS Ntrip service supports the different Ntrip protocol versions/options (Ntrip v1, Ntrip v2 over HTTP and Ntrip v2 over RTSP/RTP); in other words, compatibility with the user equipment is now ensured.

With respect to the performance supported by EDAS DGPS for precision agriculture applications, in the related article presented at the ENC 2018 ([15]), the results of the first in-field test, conducted by Topcon Agriculture and ESSP in Marchena (Seville, Spain) were already presented. Based on the obtained results (also summarized in the current paper), it was concluded that EDAS DGPS corrections could support pass-to-pass accuracies in the order of $20 \mathrm{~cm}$ in a consistent manner and with a high degree of repeatability.

In order to improve the understanding of the actual performance delivered by EDAS DGPS corrections from the point of view of precision agriculture applications, two additional tests were done in Lisbon (Portugal) and 
York (UK). The selected scenarios provide diversity with respect to the first test, especially in terms of distance from the farm to the selected EGNOS reference station $(\approx 320 \mathrm{~km}$ in York and $40 \mathrm{~km}$ in Lisbon, versus the 110 $\mathrm{km}$ baseline of the test in Seville) and also geographically. In all tests, a Topcon RTK solution has been running in parallel to the EDAS DGPS solution to provide the required reference for the post-processing of the recorded data. In summary, 9 different runs with a total of 78 passes were done in the two additional data campaigns.

Considering the results from the three tests, the pass-to-pass accuracy supported by EDAS DGPS corrections was below $10 \mathrm{~cm}$ for more than $60 \%$ of passes and below $20 \mathrm{~cm}$ for more than $85 \%$ of the passes. These figures exceed the results presented last year ([15]) and, hence, confirm that EDAS DGPS corrections can deliver pass-to-pass accuracies in the order of 10 to $20 \mathrm{~cm}$ in a consistent manner.

The stability of the results and the very good pass-to-pass accuracy levels observed in the York scenario, where baselines larger than $300 \mathrm{~km}$ were tested, deserve to be highlighted. Considering that, for the case of cereal and dry soil cultivation at least 1 meter -95th percentile- of absolute horizontal accuracy is required, based on the York results, it can be assumed that, within the area where EDAS DGPS supports sub-meter horizontal accuracies (up to $260 \mathrm{~km}$ from the selected EGNOS station ([19])), EDAS DGPS corrections can also support pass-to-pass accuracies in the order of 10-20 cm.

Such performance levels are considered to be appropriate for most cereal farm operations. In particular, the observed performance is sufficient to support the following precision agriculture applications:

- Spraying/Spreading of any crop type.

- Tilling of cereal.

- Harvesting of cereal.

To conclude, the test campaigns reported in this article, jointly performed by Topcon Agriculture and ESSP, confirm that EDAS DGPS corrections can support a wide range of precision agriculture applications and represent a real alternative for cereal farms, when located in the vicinity (at least up to $260 \mathrm{~km}$ away) of an EGNOS reference station, complementing the benefits that the EGNOS Signal-In-Space is already providing to a large number of agriculture users in Europe. In addition, the EDAS Ntrip service enables RTK positioning, and hence it can also be an alternative for the most demanding applications (when located within $40-50 \mathrm{~km}$ from the target EGNOS station).

\section{ACKNOWLEDGMENTS}

The authors would like to acknowledge the efforts done by the European Commission (EC) and the European GNSS Agency (GSA) to continuously support the EGNOS programme.

Finally, the authors would like to express their gratitude to their colleagues Héctor Pámpanas (Sogeti High Tech) and Julián Sedano (Topcon Agriculture) for the invaluable support in the elaboration of this paper.

\section{REFERENCES}

[1] EGNOS Data Access Service (EDAS) Definition Document, http://egnos-user-support.esspsas.eu/new_egnos_ops/content/egnos-sdds

[2] EGNOS Safety of Live Service Definition Document, http://egnos-user-support.esspsas.eu/new_egnos_ops/content/egnos-sdds

[3] EGNOS Open Service - Service Definition Document, http://egnos-user-support.esspsas.eu/new_egnos_ops/content/egnos-sdds 
[4] EDAS Client SW User Manual, ESSP-DRD-6977

[5] EDAS FTP Service - User Information Package

[6] EDAS SISNeT Service - User Information Package

[7] EDAS Ntrip Service- User Information Package

[8] RTCM 10403.1 Recommended standards for differential GNSS Services, Version 3.1, Radio Technical Commission for Maritime Services.

[9] Networked Transport of RTCM via Internet Protocol (Ntrip), version 2.0, Radio Technical Commission for Maritime ServicesRTCM 10402.1 Recommended Standards for Differential Navstar GPS Service, Version 2.1, Radio Technical Commission for Maritime Services.

[10] RTCM 10402.3 Recommended standards for differential GNSS Service Version 2.3, Radio Technical Commission for Maritime Services.

[11] MOPS for GPS/WAAS Airborne Equipment, RTCA DO-229, Revision D, Issued 13 December 2006.

[12] GSM specification. www.3gpp.org/specifications

[13] "EDAS (EGNOS Data Access Service): alternative source of differential GPS corrections for maritime users", Institute of Navigation (ION) GNSS 2015, E. Lacarra et al., ESSP SAS and Alberding GmbH.

[14] ISO 12188-1, Tractors and machinery for agriculture and forestry-Test procedures for positioning and guidance systems in agriculture-Part1: Dynamic testing of satellite-based positioning devices.

[15] "EDAS (EGNOS Data Access Service): Differential GPS corrections performance test with state-ofthe-art precision agriculture system”, ENC 2018, J. Vázquez et al., ESSP SAS.

[16] EGNOS User Support Website, https://egnos-user-support.essp-sas.eu/

[17] Pass to pass concept explanation, https://egnos-user-support.essp-sas.eu/new_egnos_ops/pass_to_pass

[18] EGNOS Service Notice \#018 - EDAS Ntrip Service evolution: support to HTTP-based communication,https://egnos-user-support.esspsas.eu/new_egnos_ops/sites/default/files/documents/service_notice_18.pdf

[19] "EDAS (EGNOS Data Access Service): Differential GNSS corrections for land applications", Institute of Navigation (ION) GNSS 2016, J. Vázquez et al., ESSP SAS. 